\title{
Effects of nanoparticles with hydrotropic nicotinamide on tacrolimus: permeability through psoriatic skin and antipsoriatic and antiproliferative activities
}

\author{
This article was published in the following Dove Press journal: \\ International Journal of Nanomedicine \\ 22 February 2017 \\ Number of times this article has been viewed
}

\section{Tao Wan* \\ Wenhui Pan* \\ Yueming Long \\ Kaiyue Yu \\ Sibo Liu \\ Wenyi Ruan \\ Jingtong Pan \\ Mengyao Qin \\ Chuanbin Wu \\ Yuehong $\mathrm{Xu}$}

Department of Pharmaceutics, School of Pharmaceutical Sciences, Sun Yat-sen University, Guangzhou, People's Republic of China

*These authors contributed equally to this work
Correspondence: Yuehong $\mathrm{Xu}$ Department of Pharmaceutics, School of Pharmaceutical Sciences, Sun Yat-sen University, No 132, East Out-ring Road, University Town, Guangzhou 510006 , People's Republic of China Tel/fax +86203994 3II9 Email Issxyh@mail.sysu.edu.cn
Abstract: The hybrid system based on nanoparticles (NPs) self-assembled by the conjugations of hyaluronic acid with cholesterol (HA-Chol NPs) combined with nicotinamide (NIC) for tacrolimus (FK506), ie, FK506 NPs-NIC, has been confirmed to exhibit a significant synergistic effect on FK506 permeation through and into intact skin; thus, it may be a promising approach for FK506 to effectively treat skin diseases. The aim of this study was to evaluate its potential for the treatment of psoriasis. In vitro permeation through the psoriatic skin was carried out, and the results revealed that the combination of NPs with NIC exhibited a significant synergistic effect on FK506 deposition within the psoriatic skin $\left(3.40 \pm 0.67 \mu \mathrm{g} / \mathrm{cm}^{2}\right)$ and penetration through the psoriatic skin $\left(30.86 \pm 9.66 \mu \mathrm{g} / \mathrm{cm}^{2}\right)$. The antipsoriatic activity of FK506 NPs-NIC was evaluated through the treatment for imiquimod (IMQ)-induced psoriasis. The psoriasis area and severity index (PASI) score demonstrated that FK506 HA-Chol NPs-NIC exerted the effect on ameliorating the skin lesions comparable to clobetasol propionate (a positive drug for psoriasis) and superior to commercial FK506 ointment (Protopic ${ }^{\circledR}$ ), and the histological study showed that it presented a synergistic effect on antipsoriasis after FK506 incorporation into NPs combined with NIC hydrotropic system, which might ultimately increase the therapeutic effect and minimize the systemic side effects by reducing the overall dose of FK506. RAW 264.7 cell uptake presented the enhancement of drugs delivered into cells by HA-Chol NPs-NIC. The antiproliferative activity on HaCaT cells identified that FK506 HA-Chol NPs-NIC exhibited significant inhibiting effects on $\mathrm{HaCaT}$ proliferation. The results support that the combination of HA-Chol NPs with NIC is a promising approach for FK506 for the treatment of psoriasis.

Keywords: tacrolimus, nanoparticles, nicotinamide, percutaneous delivery, psoriasis, antiproliferation

\section{Abbreviations}

ANOVA, analysis of variance; C6, coumarin 6; Chol, cholesterol; CLSM, confocal laser scanning microscope; CP, clobetasol propionate; CCTCC, China Center for Type Culture Collection; DAPI, 4',6-diamidino-2-phenylindole; DMEM, Dulbecco's Modified Eagle's Medium; DMSO, dimethyl sulfoxide; EDC, ethylcarbodiimide hydrochloride; FBS, fetal bovine serum; FDA, Food and Drug Administration; FK506, tacrolimus; HA, hyaluronic acid; HaCaT, immortalized human keratinocytes; IL-2, interleukin-2; HE, hematoxylin and eosin; HPLC, high-performance liquid chromatography; IMQ, imiquimod; MTT, 3-(4,5-dimethyl-2-thiazolyl)-2,5-diphenyl2-H-tetrazolium bromide; LSD, least significant difference; NF- $\kappa \mathrm{B}$, nuclear factor- $\kappa \mathrm{B}$; 
NHS, $N$-hydroxysuccinimide; NS, normal saline; OD, optical density; PASI, psoriasis area and severity index; SC, stratum corneum; SD, standard deviation; NIC, nicotinamide; NPs, nanoparticles; PARP-1, poly(ADP-ribose) polymerase-1; PBS, phosphate buffer solution.

\section{Introduction}

Psoriasis is one of the most common chronic immunemediated inflammatory erythematosquamous dermatoses, prevalent among $2 \%-5 \%$ of the world population. It is characterized by keratinocyte hyperproliferation, hyperplasia of the epidermis, and massive infiltration of inflammatory leukocytes in the epidermis and dermis. ${ }^{1-3}$ The clinical feature of visible disfiguration of erythematous skin lesions covered with white silvery scales at the skin surface often inflicts upon patients through psychological burdens and decreased quality of life.

Currently, the treatment of psoriasis composes of a wide range of treatment options, including topical agents (corticosteroids and vitamin D analogs), systemic administration (cyclosporine A, methotrexate, and retinoids), phototherapy (psoralen combination with ultraviolet radiation A [PUVA] and ultraviolet radiation $\mathrm{B}$ [UVB]), and biological treatment (specific siRNA). ${ }^{4-8} \mathrm{Up}$ to date, topical therapy remains a preferred treatment strategy for psoriasis because the site of action is direct. The challenge for topical treatment of psoriasis is the poor drug penetration through the psoriatic skin owing to the physiopathology of psoriasis. Psoriatic skin with the characteristics of thickening and abnormal maturation of the SC, lipid imbalance, and deficiency of natural moisturizers hinders drug penetration and limits therapeutic efficacy. ${ }^{9}$ A novel and improved percutaneous delivery system to overcome the SC barrier of psoriatic skin is vital to antipsoriatic drug for topical therapy.

Psoriasis often requires long-term and routine administration of anti-inflammatory drugs such as corticosteroids. Topical application of corticosteroids is the first-line therapeutics for psoriasis; however, its utility is likely to induce skin atrophy by inhibition of collagen synthesis and increased susceptibility to skin infection. ${ }^{10}$ Since the inflammation in psoriasis is related to an exacerbated immune response, immunosuppressant drug is a valid therapeutic strategy. FK506, a macrolide immunosuppressive drug isolated from Streptomyces tsukubaensis, has been proved to be useful in the treatment of psoriasis and psoriasis arthritis when administered systemically. ${ }^{11-14}$ Its mechanism is similar to that of cyclosporine A, a potent cyclic undecapeptide immunosuppressant, which is therapeutically effective in patients with severe psoriasis. ${ }^{15}$ Moreover,
FK506 is 10-100-fold better than cyclosporine A in inhibiting the mass production of lymphokines involved in IL-2 production, and therefore it has been widely used in organ transplantations. ${ }^{16}$ FK506 achieves immunosuppression by the inhibition of IL-2 and other immune response cytokine transfection, thereby preventing T-lymphocyte activation, ${ }^{17-19}$ while activated $\mathrm{T}$ cells are necessary for the development and persistence of psoriasis lesions. Clinical research data have indicated that systemic administration of FK506 can play an important role in the treatment of severe and recalcitrant psoriasis on the face, genitalia, intertriginous areas, and corporal plaques. ${ }^{20}$ FK506 is available commercially as an ointment formulation (Protopic ${ }^{\circledR}$ ) approved by the FDA for topical treatment of atopic dermatitis. In clinical practice, FK506 ointment is commonly used off-label in other skin diseases, including psoriasis, and FK506 was recommended in particular for the treatment of facial and intertriginous psoriasis by the American Academy of Dermatology. ${ }^{21}$ However, topical application of the commercial ointment failed to promote FK506 deposition in hyperkeratotic psoriatic plaques. ${ }^{22}$ Meanwhile, FK506 ointment is difficult to be washed off from the skin surface and may create a sticky sense and discomfort to the patient due to its greasy nature. Several reports demonstrated that topical application of FK506 ointment was only effective under occlusion or to some specific regions of the body where the skin was thinner and more permeable, such as the face and intertriginous areas. ${ }^{23-27}$ The poor efficacy of FK506 for topical therapy was attributed to its high hydrophobicity and high molecular weight (822.05 Da), which prevent it from penetrating into and through the skin. In our previous study, ${ }^{28}$ we have formulated a hybrid nanoparticle system for FK506 percutaneous delivery. The system was based on the combination of amphiphilic conjugations of HA-Chol-selfassembled NPs and hydrotropic NIC, ie, FK506 HA-Chol NPs-NIC, and it has been found that either NP carrier or NIC hydrotropy could enhance the permeation of FK506 through the intact skin, and the combination of HA-Chol NPs with NIC could synergistically enhance the permeation of FK506 into deeper epidermal and dermal layers of the skin in comparison with commercial FK506 ointment. These results supported that the hybrid system facilitated FK506 in the treatment of skin diseases.

In this study, the aim is to investigate the potential of FK506 HA-Chol NPs-NIC for the treatment of psoriasis. FK506 HA-Chol NPs-NIC was formulated by self-assembling. The murine psoriasis model was constructed by topical application of IMQ on mouse dorsal skin. In vitro permeability through the psoriatic skin of FK506 from HA-Chol NPs-NIC was 
evaluated in comparison with HA-Chol NPs, NIC complex solution, and FK506 ointment. The antipsoriatic activity of FK506 HA-Chol NPs-NIC against IMQ-induced psoriasis was assessed in comparison with FK506 NPs, FK506 NIC complex solution, FK506 ointment, and CP (a positive reference), and the effect was evaluated with the PASI score and histological examination. Finally, RAW 264.7 cells were used to assess the cellular uptake using a hydrophobic probe of $\mathrm{C} 6$ and HaCaT cells were used to evaluate the antiproliferative activity of FK506 HA-Chol NPs-NIC.

\section{Materials and methods Materials}

Cholesteryl chloroformate, $\mathrm{N}$-(3-dimethylaminopropyl)$N^{\prime}$-EDC, NHS, and pyrene were purchased from Aladdin (Shanghai, China). Sodium hyaluronate (HA, Mw $30 \mathrm{kDa}$ ) was obtained from Bloomage Freda Biopharm Co., Ltd (Shandong, China). Anhydrous ethylenediamine was purchased from Damao Reagent (Tianjin, China). Trimethylamine was obtained from Zhiyuan Reagent (Tianjin, China). NIC was a gift of Guangzhou Changlong (Guangzhou, China). FK506 was purchased from Teva Czech Industries s.r.o. (Opava-Komarov, Czech Republic). FK506 ointment (Protopic ${ }^{\circledR}$ ) was obtained from Astellas Toyama Co., Ltd. (Toyama, Japan). The product 502 super glue was purchased from Henggu Plastic Industry Co., Ltd (Zhejiang, China). A total of 5\% IMQ cream was purchased from Hubei Keyi Pharmaceutical Co., Ltd (Hubei, China). A total of $0.02 \%$ CP cream was obtained from Huarun Shunfeng Pharmaceutical Co., Ltd (Shenzhen, China). C6, penicillin, DAPI, and streptomycin were obtained from Sigma-Aldrich (St Louis, MO, USA). FBS and DMEM were purchased from Gibco (Gaithersburg, MD, USA). Other reagents were of analytical grade.

Murine RAW 264.7 macrophage cells were obtained from the Experimental Animal Center of Sun Yat-sen University (Guangzhou, China). HaCaT cells were obtained from CCTCC (Wuhan, China).

\section{Preparation and characterization of FK506 NPs}

FK506 NPs were prepared as described in our previous study.$^{28}$ In brief, amphiphilic HA-Chol conjugates were synthesized by amide bond formation of the $-\mathrm{NH}_{2}$ of cholesteryl amine and the $-\mathrm{COOH}$ of HA. The purified HA-Chol conjugates and FK506 were dispensed in 20\% (w/v) NIC solution or aqueous solution with the final FK506 concentration of $1 \mathrm{mg} / \mathrm{mL}$, and then, FK506-loaded HA-Chol NPs with NIC or without NIC were obtained by sonicating for 6 min using ultrasonic cell disruptor (JY92-2D; Ningbo Xinzhi Science and Technology Co., Ningbo, People's Republic of China). The particle size and zeta potential of FK506 NPs-NIC and FK506 NPs were determined by Nano ZS Zetasizer (Malvern Instruments, Malvern, UK), and they were $\sim 210 \mathrm{~nm}$ and $\sim 42 \mathrm{mV}$ and $\sim 240 \mathrm{~nm}$ and $\sim 43 \mathrm{mV}$, respectively. The encapsulation efficiency of FK506 NPs-NIC and FK506 NPs was $\sim 79$ and $72 \%$, respectively.

\section{Psoriasis mouse model induced by IMQ}

BALB/c mice (male, 6-8 weeks old) were purchased from the Experimental Animal Center of Sun Yat-sen University. The mice were housed under specific pathogen-free conditions at constant levels of humidity and temperature on $12 \mathrm{~h}$ light/dark cycles, provided with food and water ad libitum. All procedures performed using animals were in accordance with the Principles of Laboratory Animal Care and Use in Research published by the Chinese Ministry of Health, and the protocols were approved by the Institutional Animal Care and Use Committee of Sun Yat-sen University.

The dermatitis induced by topical application of IMQ on mouse dorsal skin closely resembles human plaque-type psoriasis lesions not only with respect to phenotypic presentation and histological characteristics but also in the development of the inflamed scaly skin lesions. ${ }^{29}$ Therefore, the murine psoriasis model was constructed in this study by topical application of IMQ on mouse dorsal skin. In brief, the mice were administrated a daily topical dose of $5 \mathrm{mg}$ IMQ with a commercially available cream (Hubei Keyi Pharmaceutical Co., Ltd) on the shaved dorsal skin for 10 consecutive days. The treated area was examined daily for skin inflammation or lesions, and the clinical PASI was scored based on the extent of erythema, scaling, and epidermal thickening of the inflammation on the dorsal region. After 10 days, the mice were sacrificed by cervical dislocation under sodium pentobarbital anesthesia and the treated dorsal skin was immediately excised. Punch biopsies of lesioned skin samples were fixed in 4\% paraformaldehyde and paraffin embedded for histological analysis to confirm the psoriasis-like skin inflammation induced by IMQ.

\section{In vitro permeation through psoriatic skin}

The experiment was conducted with Franz diffusion cell (TK-12A; Kaikai Technology Co., Ltd, Shanghai, China) under occlusive conditions with an effective contact area of $3.14 \mathrm{~cm}^{2}$ and a receptor volume of $7.5 \mathrm{~mL}$. After the psoriasis was successfully induced by IMQ as described in the 
"Psoriasis mouse model induced by IMQ" section, the mice were sacrificed by cervical dislocation under sodium pentobarbital anesthesia. The dorsal skin with psoriatic lesions was immediately excised, and the subcutaneous tissues were surgically removed; then, the treated psoriasis skin was sandwiched between the donor and receptor cells with SC facing upward into the donor. The receptor medium was NS containing $25 \%$ ethanol with respect to the sink condition for the permeation of FK506. Skin temperature was maintained at $32^{\circ} \mathrm{C} \pm 1^{\circ} \mathrm{C}$ throughout the experiment with a circulating water bath and stirred at $250 \mathrm{rpm}$. After equilibration, $500 \mu \mathrm{L}$ of FK506 HA-Chol NPs-NIC, HA-Chol NPs, and FK506 NIC complex suspension and $0.5 \mathrm{~g}$ of commercial preparation of FK506 ointment were applied onto the skin. In our preliminary experiment, FK506 in the receptor medium could not be detected until $10 \mathrm{~h}$ permeation in the groups with NIC and $12 \mathrm{~h}$ permeation in the group without NIC; thus, the receptor medium at intervals of $12,14,16,18,20$, and $24 \mathrm{~h}$ after permeation were sampled, and equal volume of fresh receptor medium was replenished after each sampling.

After $24 \mathrm{~h}$ permeation, the diffusion cells were dismantled, the residual formulations on the treated skin were carefully removed, and the skin was cut into pieces. Two volume of methanol was added to the skin pieces for extracting FK506 retained in the psoriatic skin. The extraction was performed by sonicating for $30 \mathrm{~min}$ following centrifuging for $5 \mathrm{~min}$ at 5,000 rpm. The supernatant was collected for the samples.

All the samples from the above were filtered with $0.22 \mu \mathrm{m}$ microporous nylon membrane and analyzed by HPLC. The HPLC system (1,260 series; Agilent Technologies, Santa Clara, CA, USA) consisted of quaternary pump (G1310A), degasser (G1322A), autosampler (G1329), column thermostat (CO-1000), ultraviolet (UV) detector (G1314A), and data processing software (Agilent Chem Station for LC systems). A C8 column (Grace $\left.{ }^{\circledR}, 250 \times 4.6 \mathrm{~mm}, 5 \mu \mathrm{m}\right)$ was used for FK506 analysis with water/isopropyl alcohol/ tetrahydrofuran $(5: 2: 2, \mathrm{v} / \mathrm{v} / \mathrm{v})$ as the mobile phase at a flow rate of $0.8 \mathrm{~mL} / \mathrm{min}$ at $55^{\circ} \mathrm{C}$, and the detection wavelength was set at $220 \mathrm{~nm}$.

\section{Antipsoriatic activity assessment} Treatment with different formulations of FK506

The animals were randomly divided into nine groups (nine mice per group) as follows: control group, IMQ group, IMQ-vehicle group, IMQ-NIC group, IMQ-FK506 ointment group, IMQ-FK506-NIC group, IMQ-FK506 NPs group, IMQ-FK506 NPs-NIC, and IMQ-CP group. Except that the control group (disease-free control group) was not treated with IMQ, each mouse of other eight groups was treated with IMQ as described in the "Psoriasis mouse model induced by IMQ" section to induce psoriasis, and then the animals were treated by applying different formulations onto the IMQ-induced dorsal region for 10 consecutive days. However, IMQ group was the disease model group without the treatment of other drugs. IMQ-vehicle group was treated with the vehicle containing excipients in the delivery system without FK506. IMQ-NIC group was treated with 20\% NIC solution, which was investigated to confirm whether NIC ameliorated the psoriasis symptom or not. IMQ-FK506 ointment group, IMQ-FK506-NIC group, IMQ-FK506 NPs group, and IMQ-FK506 NPs-NIC group were treated with different formulations containing $0.1 \%$ FK506 (equal to the content of FK506 in commercial ointment), and the different formulations were topically administered onto the IMQ-induced psoriasis lesions at an equal dose of $20 \mu \mathrm{g} / \mathrm{cm}^{2}$ of FK 506 in each group. CP, as an extremely potent synthetic steroid hormone approved for the management of psoriasis by FDA, ${ }^{30}$ was used as a positive control drug to exhibit ameliorating symptoms in this study, and it was topically administered once daily at a dose of $8 \mu \mathrm{g} / \mathrm{cm}^{2}$ in the IMQ-CP group. All the therapy lasted for 10 days.

After 10 days' treatment, mice were sacrificed by cervical dislocation under sodium pentobarbital anesthesia and the treated dorsal skin was immediately excised. Punch biopsies of lesional skin samples from each mouse in each group were fixed in 4\% paraformaldehyde and paraffin embedded for histological analysis.

\section{Scoring severity of skin inflammation}

To monitor and grade the severity of the psoriasis-like skin condition on days $0,2,4,6,8$, and 10 , the scoring system was based on the clinical PASI. ${ }^{5,29,31,32}$ Three parameters, including erythema, scaling, and thickening, were assigned independently on a scale from 0 to 4 as follows: 0 , none; 1 , slight; 2 , moderate; 3 , marked; 4, very marked. A scoring table with red taints was used to score the level of erythema. The cumulative score (erythema, scaling, and thickening) denoted the severity of inflammation (scale 0-12). After 10 days treatment, mice were sacrificed by cervical dislocation under sodium pentobarbital anesthesia. In addition of evaluation by PASI, the treated dorsal skin was immediately excised, and the thickness of the skinfold was measured in sextuplicate using a digital micrometer with an accuracy of $\pm 0.02 \mathrm{~mm}$ (Mitutoyo Corporation, Mitsutomo, Japan).

\section{Histopathological analysis}

The paraffin-embedded punch biopsies were sectioned longitudinally into $6 \mu \mathrm{m}$ thick sections. Then, the tissue 
sections were HE stained for histological and microarchitecture evaluation. Blinded to treatment group, epidermal thickness, an accepted end point for measuring psoriasis severity, was measured as an average of 20 random measurements of the distance from the SC to the deepest part of the epidermis. Experiments were conducted in sextuplicate, and the data were averaged to evaluate epidermal proliferation and inflammation.

\section{Cell culture}

The cells were cultured in DMEM supplemented with FBS $(10 \%, \mathrm{v} / \mathrm{v}), 100 \mathrm{IU} / \mathrm{mL}$ penicillin, and $100 \mu \mathrm{g} / \mathrm{mL}$ streptomycin in the humidified atmosphere of $95 \%$ air with $5 \% \mathrm{CO}_{2}$ at $37^{\circ} \mathrm{C}$ (Forma 3111; Thermo Fisher Scientific, Waltham, MA, USA). The medium was replenished every other day, until the monolayer cultures reached $60 \%-80 \%$ confluency. Subsequently, the culture medium was aspirated off and the cells were then washed with PBS, followed by incubation with trypsin-EDTA solution $(1 \mathrm{~mL})$ at $37^{\circ} \mathrm{C}$ for $5 \mathrm{~min}$. The trypsin was deactivated by the addition of $2 \mathrm{~mL}$ of DMEM supplemented with FBS. Then, the cell suspension was centrifuged at 1,000 rpm for $3 \mathrm{~min}$, and the resulting supernatant was removed. Eventually, $4 \mathrm{~mL}$ of fresh culture medium was added to resuspend the detached cells. The suspension was then ready to be used for experimental work.

\section{Cellular uptake of fluorescence-labeled formulations}

RAW 264.7 cells were cultured in $15 \mathrm{~mm}^{2}$ confocal dish $\left(1 \times 10^{5}\right.$ cells/well $)$ with DMEM. After cultured for $24 \mathrm{~h}$, the culture medium was removed and washed with PBS for three times. Two hundred microliter of HA-Chol NPs-NIC, HA-Chol NPs, NIC complex suspension, and aqueous suspension containing equal amount of $\mathrm{C} 6(0.1 \%, \mathrm{w} / \mathrm{v})$ were diluted by DMEM without FBS to $10 \mu \mathrm{g} / \mathrm{mL}$ and added to the dish. After culturing for $4 \mathrm{~h}$, the culture medium was removed and the cells were washed with PBS for three times. Then, the cells were fixed with $4 \%$ paraformaldehyde solution at ambient temperature for $15 \mathrm{~min}$, and the nucleus of cell was labeled by DAPI. RAW 264.7 cells without adding fluorophore were tested as a blank control group. The cellular uptake was evaluated using CLSM (LSM 710; Zeiss, Oberkochen, Germany). The excitation and emission wavelengths of C6 were 430 and $485 \mathrm{~nm}$, respectively.

\section{MTT cell proliferation assay}

Immortalized human $\mathrm{HaCaT}$ keratinocytes were used as the model of proliferating epidermal cells to test the antiproliferative efficacy of different formulations containing FK506. ${ }^{33-36}$
HaCaT cells in the log phase were seeded into 96-well plates (Corning Inc., Corning, NY, USA) with a density of $5 \times 10^{3}$ cells/well and maintained in an atmosphere of $5 \% \mathrm{CO}_{2}$ at $37^{\circ} \mathrm{C}$ for 24 h. HA-Chol NPs-NIC, HA-Chol NPs, NIC complex suspension, and aqueous suspension containing equal amount of FK506 (0.1\%, w/v) were diluted by culture medium to the final concentration at $10 \mu \mathrm{g} / \mathrm{mL}$ of FK506, and empty NPs-NIC vehicle was diluted as well to be the vehicle control. These different preparations were added to the cells cultured in 96-well microplates. Cell proliferation was assessed at different time points (6 and $18 \mathrm{~h}$ ). The cells with no formulations were designated as normal control cells, while blank control cells contained only culture medium were used for colorimetric zero adjustment. The medium was then removed, and the adhering cells were washed with PBS for three times. The culture fluid was then replaced with fresh DMEM without FBS containing $0.5 \mathrm{mg} / \mathrm{mL}$ of MTT. After incubation for $4 \mathrm{~h}$ at $37^{\circ} \mathrm{C}$, the MTT reaction medium was removed from the plates and $150 \mu \mathrm{L}$ of DMSO was added to solubilize formazan blue. The plates were shaken for $15 \mathrm{~min}$ in the dark to dissolve the formazan completely. The OD was then recorded at $490 \mathrm{~nm}$ with an ELISA Microplate Reader (Synergy H1; BioTek, Winooski, VT, USA). All tests were performed in triplicate, and results were calculated as a percentage of the values of the test wells over the corresponding normal control wells.

\section{Data analysis}

Each experiment was performed at least in triplicate. The data were given as mean \pm SD. The statistical differences between the groups used one-way ANOVA followed by LSD as post hoc analysis (SPSS Version 19.0; SPSS Inc., Chicago, IL, USA). $P<0.05$ was considered statistically significant.

\section{Results and discussion Permeability through psoriatic skin}

The proliferation and abnormal differentiation of keratinocytes of psoriasis render the psoriatic skin with the thickening of the SC and abnormal maturation of the SC, which exhibits abnormal epidermal barrier. Permeability through psoriatic skin comes closer to the clinical application than that through intact skin, which facilitates to elucidate the exact influence of drug delivery system on antipsoriatic drugs percutaneous delivery. Therefore, the psoriatic skin was used as the skin barrier to perform permeation study. The cumulative permeated amount of FK506 through psoriatic skin was plotted versus time and the retention of FK506 in the skin, as shown in Figure 1. FK506 ointment $(0.1 \%$, w/w) was taken as the reference group because there was no significant difference 
A

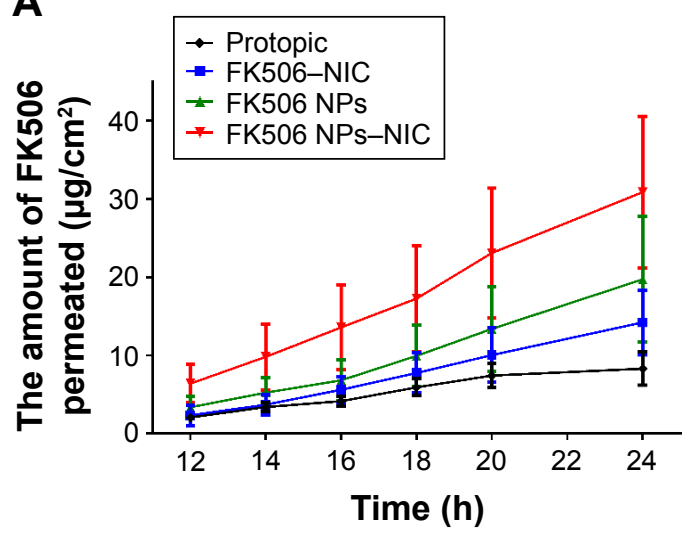

B

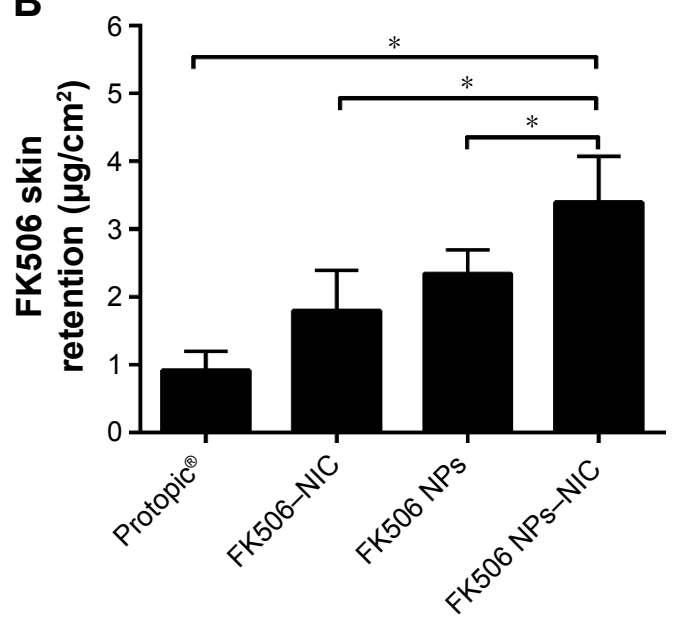

Figure I Permeation profiles of FK506 through the psoriatic skin of mouse from different formulations in vitro (A) and FK506 skin retention after 24 h permeation of different formulations in vitro (B).

Notes: Each symbol and bar represent the mean \pm SD of six determinations. Significant differences were calculated using the ANOVA test $(* P<0.05)$. The concentration of FK506 in each formulation was $1 \mathrm{mg} / \mathrm{mL}$.

Abbreviations: ANOVA, analysis of variance; FK506, tacrolimus; NIC, nicotinamide; NPs, nanoparticles; SD, standard deviation.

between FK506 ointment and 0.1\% FK506 suspension in our preliminary experiment. As shown in Figure 1, during the $24 \mathrm{~h}$ permeation, the FK506 permeation from NIC complex or HA-Chol NPs was significantly higher than that from FK506 ointment $(P<0.05)$ throughout the experiment. After $24 \mathrm{~h}$ permeation, the cumulative permeated amount of FK506 from NPs or NIC complex was 2.38-fold or 1.71-fold as that of FK506 ointment, and the skin retention of FK506 from NPs or NIC complex was 2.54-fold or 1.96-fold as that of FK506 ointment. Furthermore, FK506 from NPs-NIC showed the highest permeation at all time points in comparison with NPs or NIC complex group, and the cumulative permeated amount of FK506 was $30.86 \pm 9.66 \mu \mathrm{g} / \mathrm{cm}^{2}$ after $24 \mathrm{~h}$ permeation, which was significantly higher than that of NIC complex with $14.21 \pm 4.15 \mu \mathrm{g} / \mathrm{cm}^{2}(P<0.05)$ or NPs alone with $19.72 \pm 8.06 \mu \mathrm{g} / \mathrm{cm}^{2}(P<0.05)$, and the FK506 retention in the skin from NPs-NIC presented the highest retention of $3.40 \pm 0.67 \mu \mathrm{g} / \mathrm{cm}^{2}$ than NPs of $2.34 \pm 0.35 \mu \mathrm{g} / \mathrm{cm}^{2}$ or NIC complex of $1.80 \pm 0.59 \mu \mathrm{g} / \mathrm{cm}^{2}(P<0.05)$. The results indicated that either NPs carrier or NIC hydrotropy could enhance the permeation of FK506 through and into the psoriatic skin, and the combination of HA-Chol NPs with NIC could synergistically enhance the permeation of FK506 into and through the psoriatic skin.

As previously stated, NPs were the potential carriers for drug percutaneous delivery because they provided a sustained drug release, ie, more retention of drugs in the skin for prolonging the residence time, targeting the upper layer of the skin. ${ }^{37-39}$ In this study, NPs, NIC complex, and NPs-NIC were confirmed to enhance the permeation of
FK506 into and through the psoriatic skin. The mechanism of these systems enhancing drug permeation through psoriatic skin might be attributed to the following facts. For NPs, the encapsulating FK506 accumulated on the skin surface to create high local concentration of FK506 and then facilitated the drug's sustained release and permeation through the skin. For NIC complex, FK506 permeation was probably facilitated in two ways. First, psoriatic skin presented drier or more rigid than the intact skin, ${ }^{40}$ and NIC exhibited moisturizing effect on the skin, ${ }^{39}$ which would modify the barrier property and enhance the penetration of drugs. Second, the hydrotropic effect of NIC on FK506 increased its solubility and provided FK506 concentration gradient and partition of skin/vehicle. Taken together, the hybrid system of HA-Chol NPs combined with NIC took both advantages of NPs and NIC to enhance FK506 permeation through and retention in the psoriatic skin. The combination of HA-Chol NPs with NIC might be a promising approach of FK506 for topical antipsoriatic treatment.

\section{Effects of FK506 HA-Chol NPs-NIC on ameliorating the incrassation and inflammation of the psoriatic skin}

The skin disorders of IMQ-induced psoriasis include erythema, scales, and thickness. Two or three days after the initiation of IMQ application, the treated dorsal skin of mouse started to display typical symptoms of erythema, scaling, and thickening. The clinical PASI was scored based on the extent of erythema, scales, and thickness on days $0,2,4,6$, 8 , and 10 , and the average scores were calculated. The score 
of erythema, scales, or thickness was plotted as a function of time (Figure $2 \mathrm{~A}-\mathrm{C}$, respectively). The total score was calculated as the sum of erythema, scales, and thickness, and it was also plotted as a function of time (Figure 2D). At the time point of day 8 , representative clinical features of the mouse were photographed (Figure 3A), and the thickness of the skinfold was measured (Figure 3B). As shown in Figure 2, the severity increased with continuous application of IMQ and the PASI score peaked on day 8. After treating with different formulations containing $0.1 \%$ FK506, the symptoms were alleviated with different levels. Reduction in the PASI score indicated the degree of healing of the treatment. The PASI score of IMQ-FK506-NIC group, IMQ-FK506 NPs group, or IMQ-FK506 NPs-NIC group was significantly less than that of IMQ group $(P<0.05)$, which was comparable to IMQ-CP $(P>0.05)$ throughout the experiment. After 10 days' treatment by either of these formulations, Figure $3 \mathrm{~A}$ presents these formulations strongly ameliorating

\section{A}
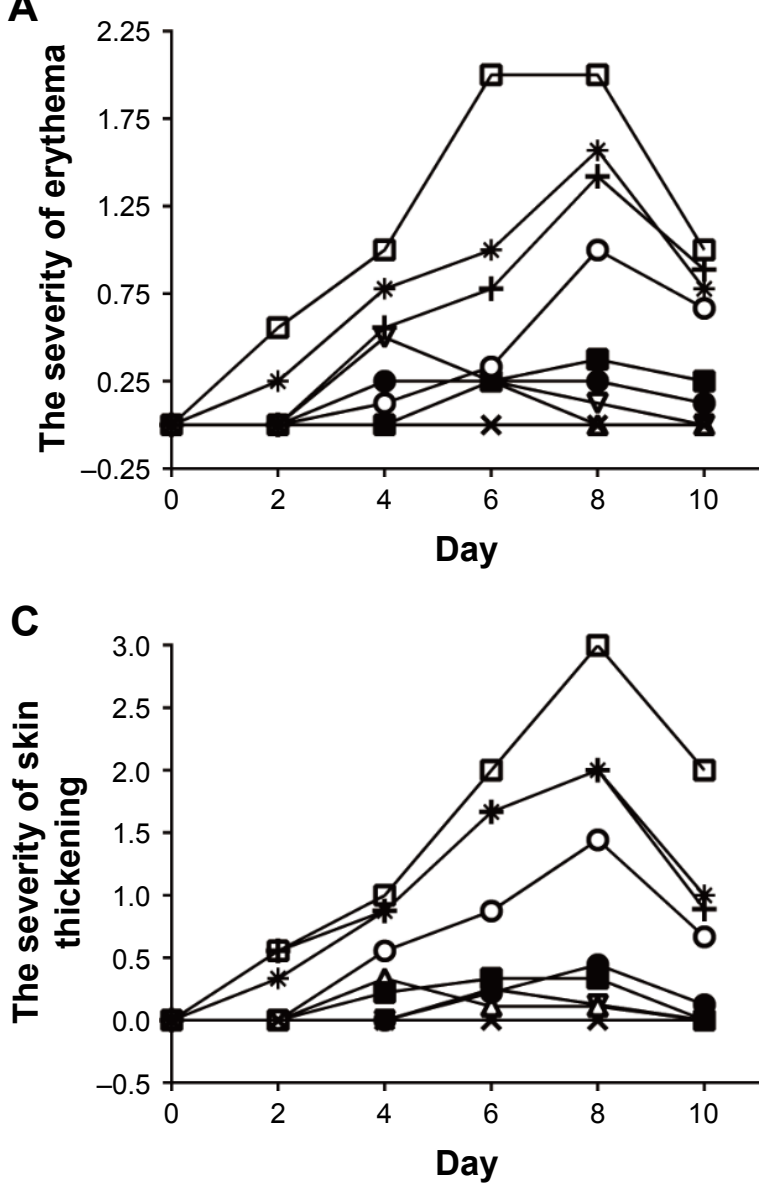

the development of psoriasis-like skin lesions. However, there was no significant difference among IMQ-FK506NIC group, IMQ-FK506 NPs group, and IMQ-FK506 NPs-NIC group in the PASI score. IMQ-FK506 ointment group also presented inhibiting the severity of IMQ-induced psoriasis in comparison with IMQ group $(P<0.05)$; however, IMQ-FK506-NIC group, IMQ-FK506 NPs group, or IMQ NPs-NIC group was significantly more effective than that of IMQ-FK506 ointment group $(P<0.05)$. The enhanced antipsoriatic effects of FK506-NIC and FK506 NPs were mainly attributed to the hydrotrope of NIC on FK506 and the nanoencapsulation of NPs on FK506, respectively. However, the visible evidence based on PASI score could not present the combined effect of FK506 NPs-NIC in comparison with FK506 NPs or FK506-NIC. The results might be attributed to the following factors. First, PASI score evaluation was based on visible signs of skin lesion, which could not identify the exact difference between the groups treated with FK506 NPs
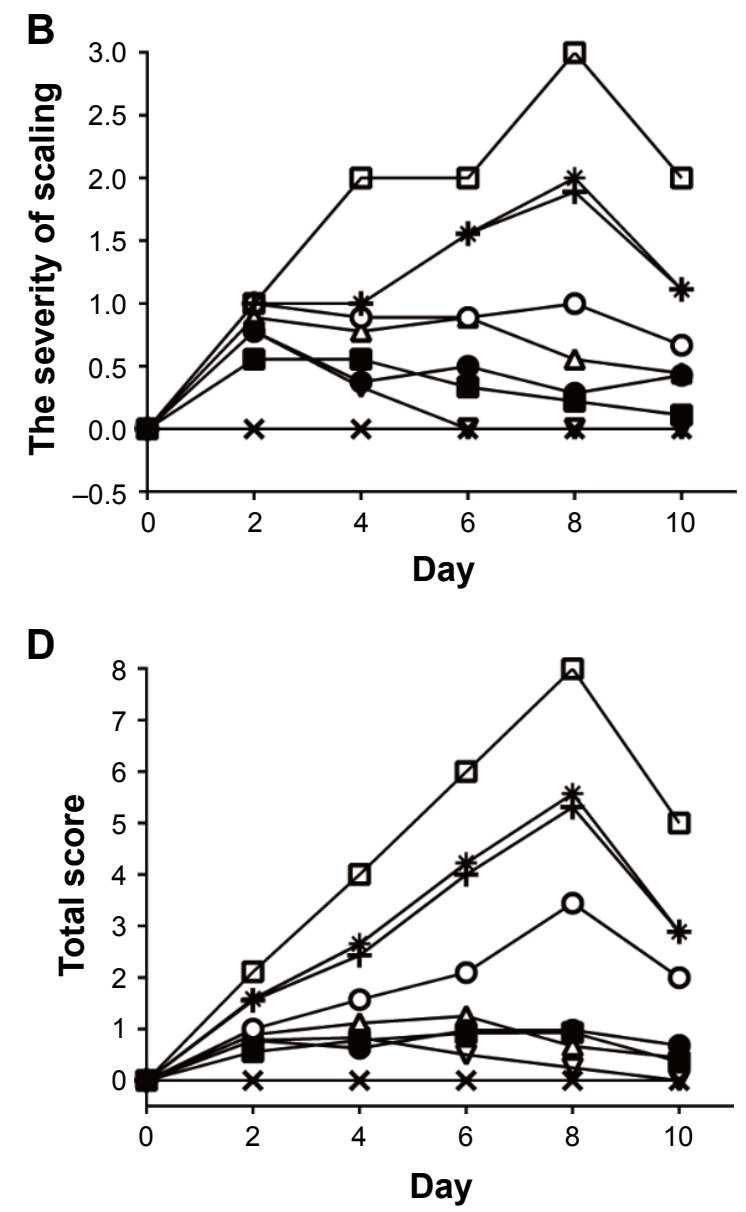

Figure 2 The severity of each psoriatic symptom; erythema (A), scaling (B), skin thickening (C), and total score (D) over time.

Notes: The severity of each psoriatic symptom was evaluated with the PASI score of psoriatic dorsal regions of mouse. Scoring was performed on days $0,2,4,6,8$, and I0, based on the erythema, scales, and thickness of PASl; each symbol represents the mean \pm SD of nine animals. Each symptom was assigned a score of $0-4$ as follows: 0 , none; I, slight;

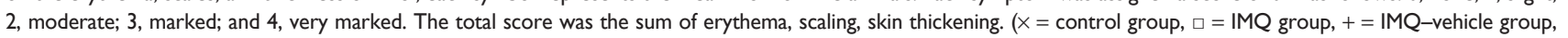
* = IMQ-NIC group, $\circ=$ IMQ-Protopic ${ }^{\circledR}$ group, $\bullet=I M Q-F K 506-N I C$ group, $=$ = IMQ-FK506 NPs group, $\Delta=I M Q-F K 506$ NPs-NIC group, and $\nabla=I M Q-C P$ group).

Abbreviations: CP, clobetasol propionate; FK506, tacrolimus; IMQ, imiquimod; NIC, nicotinamide; NPs, nanoparticles; PASI, psoriasis area severity index. 


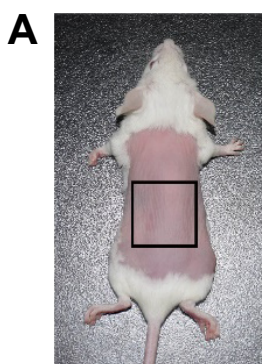

Control



IMQ-vehicle



IMQ-FK506 NPs



IMQ

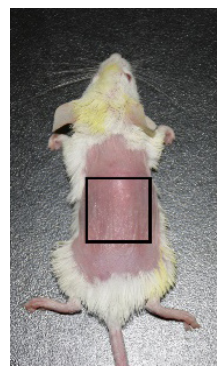

IMQ-Protopic

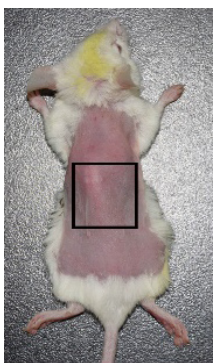

IMQ-FK506 NPs-NIC

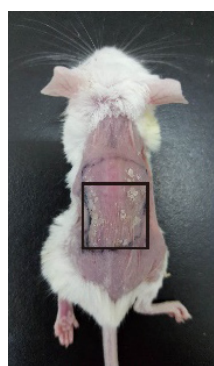

IMQ-NIC

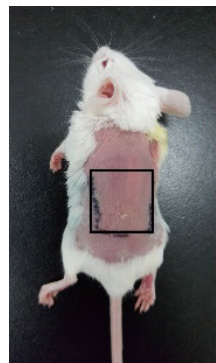

IMQ-FK506-NIC

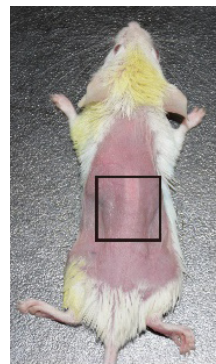

IMQ-CP
B

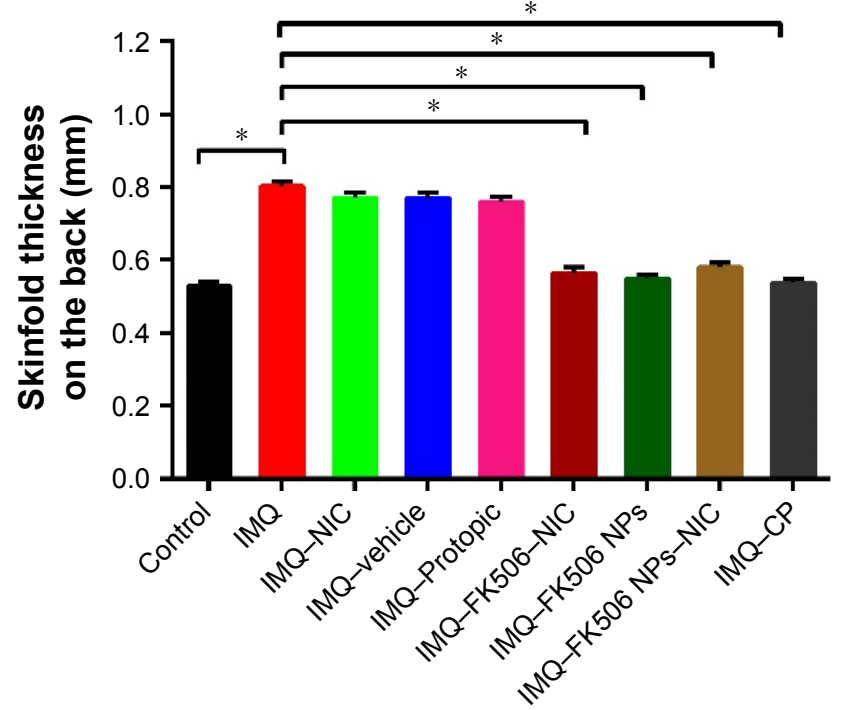

Figure 3 Representative photography of mouse after 8 days treatment with different formulations $(\mathbf{A})$ and skinfold thickness measurement (B).

Notes: Each bar represents the mean \pm SD of nine determinations. Significant differences were calculated using the ANOVA test $(* P<0.05)$. The concentration of FK506 in each formulation was $1 \mathrm{mg} / \mathrm{mL}$.

Abbreviations: ANOVA, analysis of variance; CP, clobetasol propionate; FK506, tacrolimus; IMQ, imiquimod; NIC, nicotinamide; NPs, nanoparticles; SD, standard deviation.

or FK506-NIC and FK506 NPs-NIC. Second, FK506 with $0.1 \%$ concentration after NIC solubilizing or NPs encapsulating had obtained the maximum therapeutic effect comparable to that of CP; thus, the combination of NPs with NIC might reduce the overall dose of FK506 for antipsoriasis. However, the hypothesis will be the subject to further research.

IMQ-NIC and IMQ-vehicle groups presented slight reduction in the score and a little improvement in the appearance of dorsal skin, which might be attributed to the effect of NIC. In the cosmetic industry, NIC was widely used in topical preparations due to its moisturizing and depigmenting properties. The existing preclinical data show that NIC has the potential for topical application in cosmetic or pharmaceutical industry because of its barrier restoration, anti-inflammatory, and depigmenting effects. ${ }^{41,42}$ Moreover, NIC has a therapeutic potential as an inhibitor of proinflammatory cytokines in inflammatory disease. ${ }^{43}$ Activation of NF- $\mathrm{KB}$ is a very important factor in both lesional and nonlesional skin from patients with psoriasis. NIC was an inhibitor of PARP-1, which regulated the expression of inflammatory cytokines, adhesion molecules, chemokines, and inflammatory mediators (such as interleukin-12, interleukin-23, and tumor necrosis factor- $\alpha$ ) through enhancing NF- $\kappa \mathrm{B}-$ mediated transcription. This mechanism implied that suppression of NF- $\mathrm{KB}$ was involved in NICresulted anti-inflammation, antiproliferative, and antipsoriatic effects. ${ }^{43,44}$ It was also reported that NIC may prove a useful addition to the antipsoriatic repertoire and may enhance the efficacy of calcipotriene when used in combination for topical psoriasis treatment. ${ }^{45}$ Therefore, NIC might play a dual role of hydrotrope for water-insoluble FK506 and adjuvant therapeutic agent in the hybrid system of FK506 NPs-NIC for antipsoriasis.

After 10 days' treatment, the thickness of the skinfold from the IMQ-FK506-NIC group, IMQ-FK506 NPs group, or IMQ-FK506 NPs-NIC group was thinner than that from 
the IMQ group, and comparable to that of IMQ-CP, while there was no significant difference between IMQ-NIC, IMQ-vehicle, or IMQ-Protopic ${ }^{\circledR}$ and IMQ groups. Taken together, FK506-NIC, FK506 NPs, or FK506 NPs-NIC can significantly ameliorate the visible inflammation signs of IMQ-induced psoriasis.

\section{Histological analysis}

The HE staining of dorsal skin was performed to observe the histological changes of the skin induced with IMQ and then treated with different formulations. As shown in Figure 4, when the dorsal skin was induced by IMQ, it presented an increased epidermal hyperplasia, elongation of rete-like ridges, acanthosis in the epidermis, and perivascular infiltration of the inflammatory cells in the upper dermis, resembling closely a phenotype typical of human psoriatic skin. Additionally, abnormal keratinocyte differentiation with marked hyperkeratosis was observed. The hyperplasia of basal and suprabasal keratinocytes resulted in epidermal thickening. The epidermal thickness of each group was measured in microscope (Figure 5). The epidermal thickness of the control group without any treatment was $16.1 \pm 4.24 \mu \mathrm{m}$, while the epidermal thickness after IMQ-induced was $88.7 \pm 15.90 \mu \mathrm{m}$, and that of IMQ-NIC, IMQ-vehicle, or IMQ-Protopic ${ }^{\circledR}$ treatment was $73.5 \pm 8.70,69.6 \pm 10.36$, or $57.1 \pm 12.51 \mu \mathrm{m}$, respectively. IMQ-FK506 ointment presented a reduction in epidermal thickness, which confirmed that FK506 was effective in antipsoriasis; however, the effect needed to be improved. The epidermal thickness was significantly reduced


IMQ-FK506 NPs group with 26.4 $\pm 6.09 \mu \mathrm{m}(P<0.05)$, IMQFK506 NPs-NIC group with 20.6 $\pm 7.13 \mu \mathrm{m}(P<0.05)$, and IMQ-CP group with $21.7 \pm 6.32 \mu \mathrm{m}(P<0.05)$. The reduction of effect on epidermal thickening was in the order of FK506 NPs-NIC $\approx$ CP $>$ FK506-NIC $\approx$ FK506 NPs. Compared to FK506-NIC or FK506 NPs, FK506 NPs-NIC presented a synergistic effect on antipsoriasis and comparable to that of $\mathrm{CP}$. The histological study provided further evidence that FK506 NPs-NIC ameliorated IMQ-induced psoriasis and presented a synergistic effect on antipsoriasis after FK506 incorporation into NPs combined with NIC hydrotropic system.

Based on the antipsoriatic activity, HA-Chol NPs-NIC was supposed to reduce the overall dose and drug consumption of FK506, by effectively solubilizing, encapsulating, and localizing at the applied target site and will ultimately increase the therapeutic effect and minimize the systemic side effects.

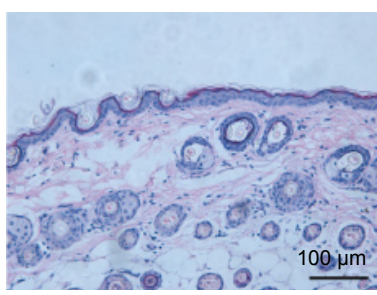

Control

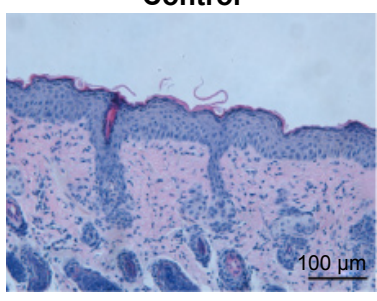

IMQ-vehicle

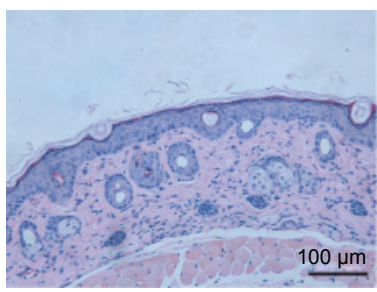

IMQ-FK506 NPs

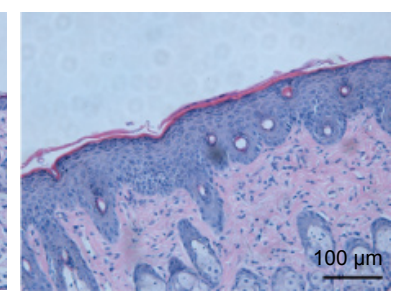

IMQ

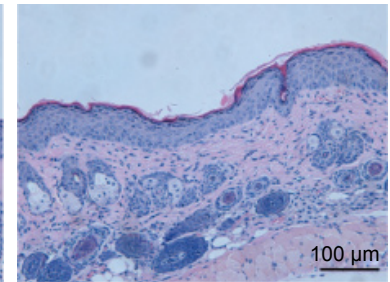

IMQ-Protopic

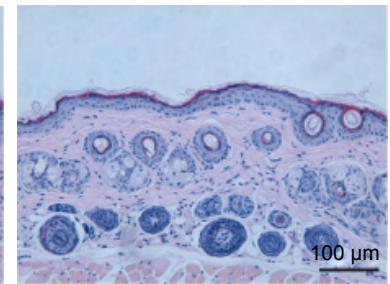

IMQ-FK506 NPs-NIC

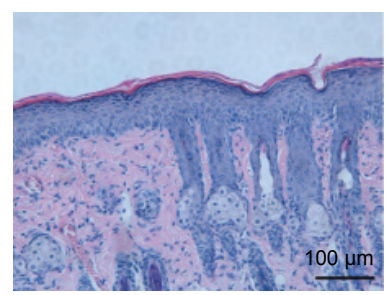

IMQ-NIC

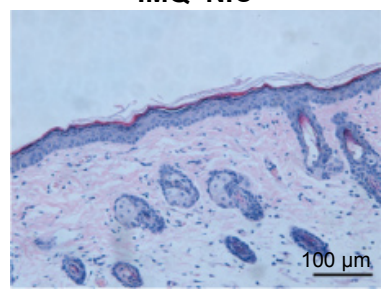

IMQ-FK506-NIC

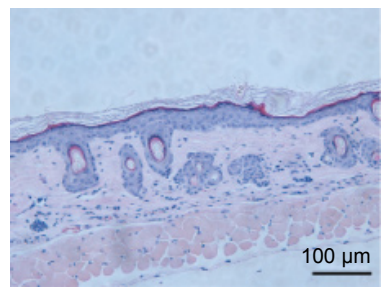

IMQ-CP

Figure 4 Histological analysis of skin after treatment with different formulations.

Notes: The epidermal thickness was used to assess the epidermal hyperplasia. The mean of epidermal thickness was calculated based on 20 random site measurements. The concentration of FK506 in each formulation was $1 \mathrm{mg} / \mathrm{mL}$. Scale bar: $100 \mu \mathrm{m}$.

Abbreviations: ANOVA, analysis of variance; CP, clobetasol propionate; FK506, tacrolimus; IMQ, imiquimod; NIC, nicotinamide; NPs, nanoparticles. 


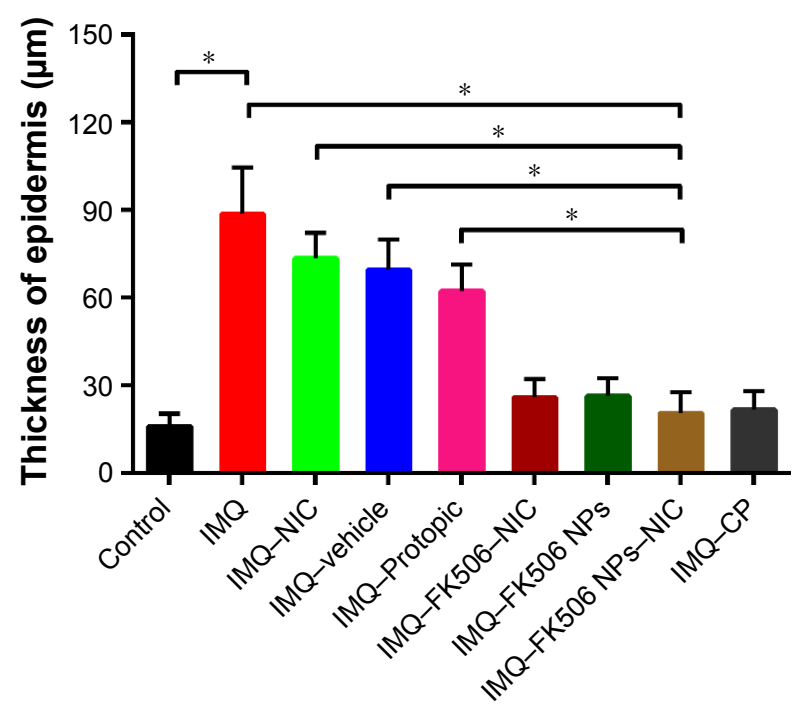

Figure 5 The epidermal thickness measured under the microscope. Notes: The epidermal thickness was used to assess the epidermal hyperplasia. The mean of epidermal thickness was calculated based on 20 random site measurements. Significant differences were calculated using the ANOVA test $(* P<0.05)$. The concentration of FK506 in each formulation was $1 \mathrm{mg} / \mathrm{mL}$.

Abbreviations: ANOVA, analysis of variance; CP, clobetasol propionate; FK506, tacrolimus; IMQ, imiquimod; NIC, nicotinamide; NPs, nanoparticles.

\section{Cellular uptake of C6-loaded NPs into RAW 264.7}

Psoriasis is an immune-mediated inflammatory skin disease; since macrophages and dendritic cells play key roles in the immune response, delivery of FK506 to these cells would facilitate the immunotherapy. Therefore, cellular uptake of drugs by murine macrophages was investigated. We chose RAW 264.7 cell to evaluate the cellular uptake of HA-Chol NPs. C6 was used as the hydrophobic fluorescence probe to mimic the hydrophobic drug, and the cellular uptake was observed by CLSM. The uptake of C6 by RAW 264.7 cell from HA-Chol NPs-NIC, HA-Chol NPs, $20 \%$ (w/v) NIC solution, and aqueous suspension containing equal amount of C6 $(10 \mu \mathrm{g} / \mathrm{mL})$ was evaluated (Figure 6). As shown in Figure 6A, RAW 264.7 cells presented the nucleus labeled by DAPI in the DMEM group without background green fluorescence interference. After $4 \mathrm{~h}$ incubation, Figure 6B presents negligible fluorescence in the $\mathrm{C} 6$ suspension group indicating very little C6 uptake by RAW 264.7 cells, and Figure $6 \mathrm{C}$ shows that NIC solution could slightly enhance the cell uptake of $\mathrm{C} 6$ compared to that of $\mathrm{C} 6$ suspension. However, when C6 was loaded in HA-Chol NPs, the fluorescence intensity was significantly strengthened, indicating that its uptake by RAW 264.7 cells was significantly enhanced (Figure 6D). When C6 was loaded in HA-Chol NPs with NIC, the fluorescence intensity was a little stronger than that of HA-Chol NPs alone (Figure 6E). The results of the cellular uptake by RAW 264.7 indicated that the system of HA-Chol NPs with NIC facilitated to deliver the drug to immune cell and to exert an effect on immune-mediated skin disease.

\section{Antiproliferative effect of different formulations}

Psoriasis was mainly characterized by an aberrant hyperproliferation of keratinocyte in the interfollicular epidermis. ${ }^{46}$ Inhibition of epidermal cell proliferation is very important in the treatment of psoriasis. To evaluate the inhibiting effect of different formulations on $\mathrm{HaCaT}$ cell proliferation, cell viability was measured with time. As shown in Figure 7, after incubation with different formulations for $6 \mathrm{~h}$, no cytotoxicity was observed in the cells treated with NPs-NIC vehicle. Both FK506-NIC and FK506 NPs-NIC significantly delayed the growth of $\mathrm{HaCaT}$ in comparison with the control group indicating the antiproliferative effects on $\mathrm{HaCaT}$, while the other groups containing the equal amount of FK506 (0.1\%) such as FK506 suspension and FK506 NPs presented no significant inhibiting effects on $\mathrm{HaCaT}$. Based on these comparisons, the inhibiting effects of FK506-NIC and FK506 NPs-NIC on HaCaT growth under $6 \mathrm{~h}$ incubation are mainly attributed to the solubilization of NIC on FK506, which ensured the efficient concentration of FK506. While FK506 was encapsulated in NPs, it released in a sustained manner from NPs; therefore, FK506 was under the efficient concentration. However, after $18 \mathrm{~h}$ incubation, the formulations including FK506-NIC, FK506 NPs, FK506 NPs-NIC, and FK506 suspension exhibited significantly inhibiting effects on $\mathrm{HaCaT}$ growth with the cell viability of $53 \% \pm 5 \%, 83 \% \pm 6 \%, 61 \% \pm 7 \%$, and $83 \% \pm 13 \%$, respectively. Combined the results from 6 and $18 \mathrm{~h}$, it can be concluded that FK506 exerts the inhibiting effect on $\mathrm{HaCaT}$ growth in a time-dependent manner. Interestingly, NPs-NIC vehicle also inhibited the growth after incubation for $18 \mathrm{~h}$, and the cell viability of FK506-NIC group decreased more than that of the other group. These results may be attributed to the high solubility of FK506 with the NIC hydrotropy and that NIC itself can promote the antiproliferative effect. Indeed, our preliminary studies showed that NIC markedly impaired the proliferation and differentiation of $\mathrm{HaCaT}$ cell line. As expected, HA-Chol NPs-NIC showed a strong inhibitory effect on the proliferation of $\mathrm{HaCaT}$ cells. However, there was no significant difference between the HA-Chol NPs-NIC group and the FK506-NIC group. Overall, these results show that FK506 HA-Chol NPs-NIC could efficiently inhibit the proliferation of $\mathrm{HaCaT}$ and have a promising prospect in the treatment of psoriasis. 


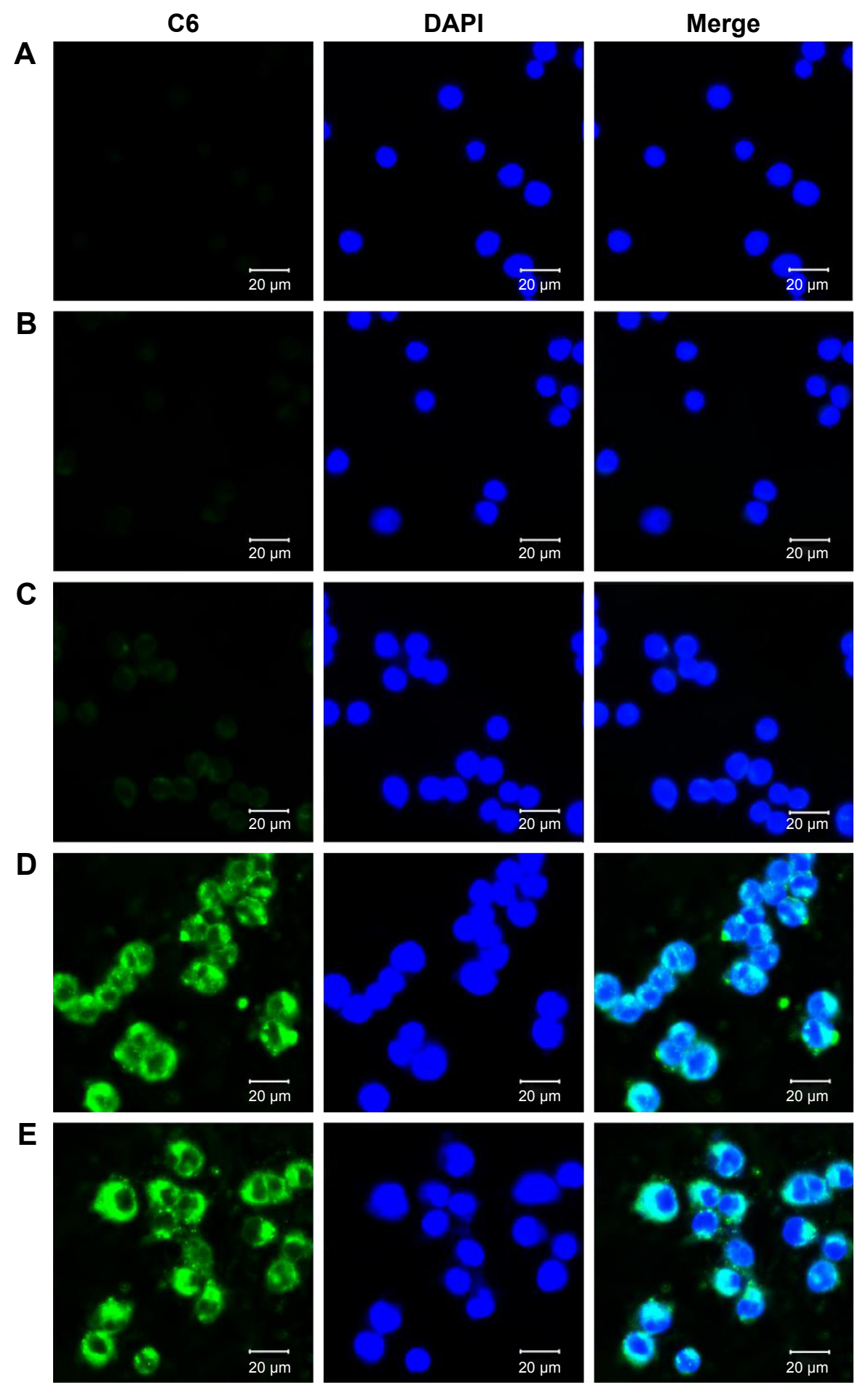

Figure 6 Confocal laser scanning microscopy of cell uptake by RAW 264.7 cell after $4 \mathrm{~h}$ administration of DMEM (A), C6 suspension (B), C6 NIC complex suspension (C), C6 HA-Chol NPs suspension (D), and C6 HA-Chol NPs-NIC suspension (E).

Notes: The concentration of $\mathrm{C} 6$ in each formulation was $10 \mu \mathrm{g} / \mathrm{mL}$. Cells were stained for nuclei (in blue) and C6 (in green). Scale bar: $20 \mu \mathrm{m}$. Magnification $\times 630$.

Abbreviations: C6, coumarin 6; Chol, cholesterol; DAPI, 4',6-diamidino-2-phenylindole; DMEM, Dulbecco's Modified Eagle's Medium; HA, hyaluronic acid; NIC, nicotinamide; NPs, nanoparticles.

\section{Conclusion}

The hybrid system of HA-Chol NPs combination with NIC for FK506 has been confirmed to exhibit a significant synergistic effect on FK506 permeation through and into the intact skin; therefore, it may be a promising approach for FK506 to effectively treat skin diseases. In this study, in vitro permeation through the psoriatic skin indicated that either NIC or HA-Chol NPs alone significantly enhanced FK506 


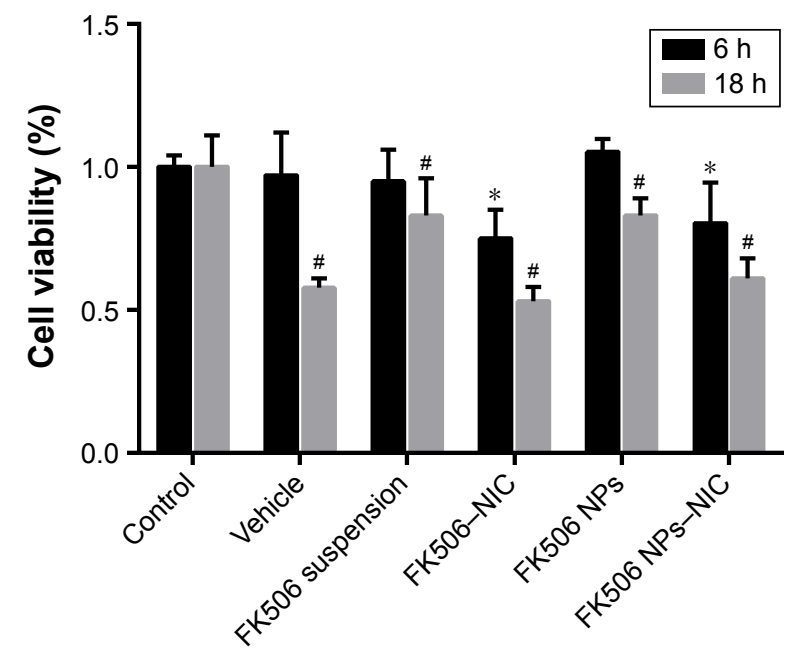

Figure 7 Antiproliferation effects of different formulations on $\mathrm{HaCaT}$ cells. Notes: Each symbol and bar represent the mean \pm SD of three determinations. Significant differences were calculated using the ANOVA test $(* P<0.05$, compared to $6 \mathrm{~h}$ control and ${ }^{\# P}<0.05$ compared to $18 \mathrm{~h}$ control). The concentration of FK506 in each formulation was $1 \mathrm{mg} / \mathrm{mL}$.

Abbreviations: ANOVA, analysis of variance; FK506, tacrolimus; SD, standard deviation; NIC, nicotinamide; NPs, nanoparticles.

permeation through and into the psoriatic skin, and the combination of HA-Chol NPs with NIC exhibited a significant synergistic effect on the permeation in comparison with NIC or HA-Chol NPs alone. The treatment for IMQ-induced psoriasis demonstrated that FK506 HA-Chol NPs-NIC had an ameliorating effect on the skin lesions comparable to that of CP and superior to that of FK506 ointment and that it presented a synergistic effect on antipsoriasis after FK506 incorporation into NPs combined with NIC hydrotropic system, which might ultimately increase the therapeutic effect and minimize systemic side effects by reducing the overall dose and consumption of FK506. RAW 264.7 cell uptake presented the enhancement of drugs delivered into cells by HA-Chol NPs-NIC. The antiproliferative activity on HaCaT cells identified that FK506 HA-Chol NPs-NIC exhibited significant inhibiting effects on $\mathrm{HaCaT}$ proliferation. In conclusion, the results indicate that the combination of HA-Chol NPs with NIC is a promising approach for FK506 for the treatment of psoriasis. Nevertheless, further works on the reduction of FK506 dose and the immunerelated molecular mechanism of antipsoriasis need to be carried out to clarify the potential of HA-Chol NPs-NIC for FK506 as a percutaneous delivery system for the treatment of psoriasis.

\section{Acknowledgment}

The study was financially supported by the National Natural Science Foundation of China (nos 81073066 and 81473358).

\section{Disclosure}

The authors report no conflicts of interest in this work.

\section{References}

1. Nickoloff BJ, Nestle FO. Recent insights into the immunopathogenesis of psoriasis provide new therapeutic opportunities. J Clin Invest. 2004; 113(12):1664-1675.

2. Hamminga EA, van der Lely AJ, Neumann HA, Thio HB. Chronic inflammation in psoriasis and obesity: implications for therapy. Med Hypotheses. 2006;67(4):768-773.

3. Nestle FO, Kaplan DH, Barker J. Psoriasis. N Engl J Med. 2009; 361(5):496-509.

4. Gustafson CJ, Watkins C, Hix E, Feldman SR. Combination therapy in psoriasis: an evidence-based review. Am J Clin Dermatol. 2013; 14(1): 9-25.

5. Jiang W, Zhu FG, Bhagat L, et al. A Toll-like receptor 7, 8, and 9 antagonist inhibits Th1 and Th17 responses and inflammasome activation in a model of IL-23-induced psoriasis. J Invest Dermatol. 2013;133(7): $1777-1784$.

6. Boakye CH, Patel K, Doddapaneni R, Bagde A, Marepally S, Singh M. Novel amphiphilic lipid augments the co-delivery of erlotinib and IL36 siRNA into the skin for psoriasis treatment. $J$ Control Release. Epub 2016 May 8.

7. Depieri LV, Borgheti-Cardoso LN, Campos PM, et al. RNAi mediated IL-6 in vitro knockdown in psoriasis skin model with topical siRNA delivery system based on liquid crystalline phase. Eur $J$ Pharm Biopharm. 2016;105:50-58.

8. Desmet E, Bracke S, Forier K, et al. An elastic liposomal formulation for RNAi-based topical treatment of skin disorders: proof-of-concept in the treatment of psoriasis. Int J Pharm. 2016;500(1-2):268-274.

9. Sala M, Elaissari A, Fessi H. Advances in psoriasis physiopathology and treatments: up to date of mechanistic insights and perspectives of novel therapies based on innovative skin drug delivery systems (ISDDS). J Control Release. 2016;239:182-202.

10. Schoepe S, Schäcke H, May E, Asadullah K. Glucocorticoid therapyinduced skin atrophy. Exp Dermatol. 2006;15(6):406-420.

11. Honbo T, Kobayashi M, Hane K, Hata T, Ueda Y. The oral dosage form of FK-506. Transplant Proc. 1987;19(5 suppl 6):17-22.

12. Kino T, Hatanaka H, Hashimoto M, et al. FK-506, a novel immunosuppressant isolated from a Streptomyces. I. Fermentation, isolation, and physico-chemical and biological characteristics. J Antibiot. 1987; 40(9):1249-1255.

13. Lythgoe M, Abraham S. Tacrolimus: an effective treatment in refractory psoriatic arthritis following biologic failure. Clin Exp Rheumatol. 2016; 34(1 suppl 95):12-13.

14. Yoon KH. Successful usage of tacrolimus (FK506) in resistant/relapsed rheumatic diseases. APLAR. J Rheumatol. 2004;7:44-48.

15. Thomson AW. FK-506 - how much potential? Immunol Today. 1989; 10(1):6-9.

16. Tocci MJ, Matkovich DA, Collier KA, et al. The immunosuppressant FK506 selectively inhibits expression of early $\mathrm{T}$ cell activation genes. J Immunol. 1989;143(2):718-726.

17. Thomson AW, Bonham CA, Zeevi A. Mode of action of tacrolimus (FK506): molecular and cellular mechanisms. Ther Drug Monit. 1995; 17(6):584-591.

18. Woo DK, James WD. Topical tacrolimus: a review of its uses in dermatology. Dermatitis. 2005;16(1):6-21.

19. Goebel AS, Neubert RH, Wohlrab J. Dermal targeting of tacrolimus using colloidal carrier systems. Int J Pharm. 2011;404(1-2):159-168.

20. Bos JD, Witkamp L, Zonnevald IM, et al. Systemic tacrolimus (FK 506) is effective for the treatment of psoriasis in a double-blind, placebocontrolled study. Arch Dermatol. 1996;132(4):419-423.

21. American Academy of Dermatology [webpage on the Internet]. Psoriasis: Recommendations for Topical Tacrolimus and Pimecrolimus. Available from: https://www.aad.org/practice-tools/quality-care/ clinical-guidelines/psoriasis/topical-therapy/recommendations-fortopical-tacrolimus-and-pimecrolimus. Accessed January 5, 2017. 
22. Zonneveld IM, Rubins A, Jablonska S, et al. Topical tacrolimus is not effective in chronic plaque psoriasis - a pilot study. Arch Dermatol. 1998; 134(9):1101-1102.

23. Remitz A, Reitamo S, Erkko P, Granlund H, Lauerma AI. Tacrolimus ointment improves psoriasis in a microplaque assay. $\mathrm{Br} J$ Dermatol. 1999;141(1):103-107.

24. Freeman AK, Linowski GJ, Brady C, et al. Tacrolimus ointment for the treatment of psoriasis on the face and intertriginous areas. J Am Acad Dermatol. 2003;48(4):564-568.

25. Lebwohl M, Freeman AK, Chapman MS, et al; Tacrolimus Ointment Study Group. Tacrolimus ointment is effective for facial and intertriginous psoriasis. J Am Acad Dermatol. 2004;51(5):723-730.

26. Kroft EB, Erceg A, Maimets K, Vissers W, van der Valk PG, van de Kerkhof PC. Tacrolimus ointment for the treatment of severe facial plaque psoriasis. J Eur Acad Dermatol Venereol. 2005;19(2): 249-251.

27. Martin Ezquerra G, Sanchez Regana M, Herrera Acosta E, Umbert Millet P. Topical tacrolimus for the treatment of psoriasis on the face, genitalia, intertriginous areas and corporal plaques. $J$ Drugs Dermatol. 2006;5(4):334-336.

28. Pan W, Qin M, Zhang G, et al. Combination of hydrotropic nicotinamide with nanoparticles for enhancing tacrolimus percutaneous delivery. Int J Nanomedicine. 2016;11:4037-4050.

29. van der Fits L, Mourits S, Voerman JS, et al. Imiquimod-induced psoriasis-like skin inflammation in mice is mediated via the IL-23/ IL-17 axis. J Immunol. 2009;182(9):5836-5845.

30. Sun J, Dou W, Zhao Y, Hu J. A comparison of the effects of topical treatment of calcipotriol, camptothecin, clobetasol and tazarotene on an imiquimod-induced psoriasis-like mouse model. Immunopharmacol Immunotoxicol. 2014;36(1):17-24.

31. Qin S, Wen J, Bai XC, et al. Endogenous n-3 polyunsaturated fatty acids protect against imiquimod-induced psoriasis-like inflammation via the IL-17/IL-23 axis. Mol Med Rep. 2014;9(6):2097-2104.

32. Ueyama A, Yamamoto M, Tsujii K, et al. Mechanism of pathogenesis of imiquimod-induced skin inflammation in the mouse: a role for interferonalpha in dendritic cell activation by imiquimod. J Dermatol. 2014; 41(2):135-143.

33. An J, Li Z, Dong Y, Ren J, Huo J. Amentoflavone protects against

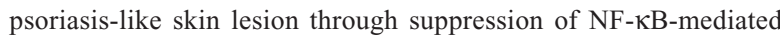
inflammation and keratinocyte proliferation. Mol Cell Biochem. 2016; 413(1-2):87-95.
34. Guichard A, Humbert P, Tissot M, Muret P, Courderot-Masuyer C, Viennet C. Effects of topical corticosteroids on cell proliferation, cell cycle progression and apoptosis: in vitro comparison on $\mathrm{HaCaT}$. Int J Pharm. 2015;479(2):422-429.

35. Chorachoo J, Saeloh D, Srichana T, et al. Rhodomytrone as a potential anti-proliferative and apoptosis inducing agent in $\mathrm{HaCaT}$ keratinocyte cells. Eur J Pharmacol. 2016;772:144-151.

36. Zhao Y, Sun J, Dou W, Hu JH. Curcumin inhibits proliferation of interleukin-22-treated HaCaT cells. Int J Clin Exp Med. 2015;8(6): 9580-9584.

37. Alvarez-Román R, Naik A, Kalia YN, Guy RH, Fessi H. Skin penetration and distribution of polymeric nanoparticles. $J$ Control Release. 2004;99(1):53-62.

38. Campbell CS, Contreras-Rojas LR, Delgado-Charro MB, Guy RH. Objective assessment of nanoparticle disposition in mammalian skin after topical exposure. J Control Release. 2012;162(1):201-207.

39. Jenning V, Gysler A, Schäfer-Korting M, Gohla SH. Vitamin A loaded solid lipid nanoparticles for topical use: occlusive properties and drug targeting to the upper skin. Eur J Pharm Biopharm. 2008;49(3): 211-218.

40. Hoffman MB, Hill D, Feldman SR. Current challenges and emerging drug delivery strategies for the treatment of psoriasis. Expert Opin Drug Deliv. 2016;13(10):1461-1473.

41. Soma Y, Kashima M, Imaizumi A, Takahama H, Kawakami T, Mizoguchi M. Moisturizing effects of topical nicotinamide on atopic dry skin. Int J Dermatol. 2005;44(3):197-202.

42. Wohlrab J, Kreft D. Niacinamide - mechanisms of action and its topical use in dermatology. Skin Pharmacol Physiol. 2014;27(6):311-315.

43. Ungerstedt JS, Blömback M, Söderström T. Nicotinamide is a potent inhibitor of proinflammatory cytokines. Clin Exp Immunol. 2003; 131(1):48-52.

44. Virág L, Szabó C. The therapeutic potential of poly (ADP-ribose) polymerase inhibitors. Pharmacol Rev. 2002;54(3):375-429.

45. Cui Y, Xing C, Ran Y. Molecular dynamics simulations of hydrotropic solubilization and self-aggregation of nicotinamide. J Pharm Sci. 2010; 99(7):3048-3059.

46. Palombo R, Savini I, Avigliano L, et al. Luteolin-7-glucoside inhibits IL-22/STAT3 pathway, reducing proliferation, acanthosis, and inflammation in keratinocytes and in mouse psoriatic model. Cell Death Dis 2016;7(8):e2344.
International Journal of Nanomedicine

\section{Publish your work in this journal}

The International Journal of Nanomedicine is an international, peerreviewed journal focusing on the application of nanotechnology in diagnostics, therapeutics, and drug delivery systems throughout the biomedical field. This journal is indexed on PubMed Central, MedLine, CAS, SciSearch $®$, Current Contents $\AA /$ Clinical Medicine,

\section{Dovepress}

Journal Citation Reports/Science Edition, EMBase, Scopus and the Elsevier Bibliographic databases. The manuscript management system is completely online and includes a very quick and fair peer-review system, which is all easy to use. Visit http://www.dovepress.com/ testimonials.php to read real quotes from published authors. 